\title{
Variations
}

Variations

Revue internationale de théorie critique

$23 \mid 2020$

Pour une gauche érotique

\section{La Théorie critique n'a pas dit son dernier mot (2004)}

Jean-Marie Vincent

\section{(2) OpenEdition}

Journals

Édition électronique

URL : http://journals.openedition.org/variations/1697

DOI : 10.4000/variations. 1697

ISSN : 1968-3960

Éditeur

Les amis de Variations

Référence électronique

Jean-Marie Vincent, «La Théorie critique n'a pas dit son dernier mot (2004) », Variations [En ligne], 23 |

2020, mis en ligne le 01 septembre 2020, consulté le 07 septembre 2020. URL : http://

journals.openedition.org/variations/1697 ; DOI : https://doi.org/10.4000/variations.1697

Ce document a été généré automatiquement le 7 septembre 2020

Les ami•e•s de Variations 


\title{
La Théorie critique n'a pas dit son dernier mot (2004)
}

\author{
Jean-Marie Vincent
}

1 L'Institut de recherche en science sociale (Institut für Sozialforschung, IfS) de Francfort a été fondé en février 1923, mais le véritable acte de naissance de ce que l'on appellera plus tard l'Ecole de Francfort date de la nomination de Max Horkheimer à la tête de l'Institut, et de son programme de février 1931 : « La situation actuelle de la philosophie sociale et les tâches d'un Institut de recherches sociales. » Il y trace les grandes lignes de recherches empiriques des- tinées à renouveler les problématiques du marxisme, souvent trop économistes et d'un déterminisme trop optimiste à ses yeux. Horkheimer, impressionné par la défaite de la révolution allemande et par les défaillances du mouvement ouvrier face à la montée du nazisme, veut qu'on s'interroge sur la conscience de classe des ouvriers et des employés. Il est en fait persuadé qu'on ne peut postuler un développement linéaire de la conscience de classe vers une conscience révolutionnaire, parce que l'économie psychique des individus de la société capitaliste ne s'y prête absolument pas. Elle est en effet profondément marquée par les difficultés récurrentes dans les processus de socialisation et les contra- dictions qu'ils recèlent. Pour comprendre la société, on ne peut donc se contenter de faire la critique de l'économie politique, il faut aussi étudier les rapports sociaux et leurs effets sur le psychisme, c'est-à-dire faire de la sociologie et de la psychologie sociale en procédant à des recherches empiriques. Toutefois, il ne peut être question de reprendre telles quelles les méthodes et les techniques habituelles dans les sciences sociales. Les recherches empiriques à effectuer doivent être éclairées et critiquées par des questionnements théoriques, par ce que Horkheimer appelle la philosophie sociale. Le terme signifie que les questionnements ne peuvent pas être purement philosophiques, mais doivent correspondre à une véritable confrontation entre philosophie et sociologie, à un va-et-vient de l'une à l'autre, pour déterminer les conditions d'une transformation de la société.

2 En effet, la philosophie purement philosophique est impuissante, alors que les sciences sociales refermées sur elles-mêmes restent à la superficie de la société, sans médiatiser 
les évidences qui se donnent pour immédiates. Une des premières évidences qu'il faut remettre en question est celle du caractère plein et définitivement constitué de l'individu. Pour Horkheimer, les individus s'illusionnent sur leur autonomie et sur leur mode d'agrégation aux rapports sociaux, sur les relations qu'ils ont les uns aux autres, et sur les relations qu'ils entretiennent avec eux-mêmes. C'est pourquoi il juge indispensable d'intégrer les apports de la psychanalyse à la recherche en sciences sociales, non parce que ce serait une psychologie des profondeurs, mais parce qu'elle peut permettre de mieux cerner ce qu'il peut y avoir d'autonomie et d'hétéronomie dans les conduites. Elle est en particulier d'une aide précieuse pour déceler pourquoi des individus et des groupes sociaux agissent à l'encontre de leurs intérêts matériels et surtout de leurs intérêts à obtenir plus de liberté et d'autonomie.

3 Sur le plan théorique, Horkheimer et ses collaborateurs - notamment Erich Fromm croient trouver une réponse dans la mise au point d'une psychologie sociale matérialiste qui, à partir des phases de la sexualité infantile, s'efforcerait de saisir les rapports à l'autorité. Sur le plan empirique, des enquêtes comparatives doivent montrer en conséquence comment la socialisation familiale, suivant les groupes sociaux et les individus, structure les relations aux autorités. De fait les enquêtes réalisées par l'Institut, pour l'essentiel dans l'émigration, montrent l'importance des syndromes autoritaires dans les couches ouvrières - manifestations de violence dans l'éducation des enfants, faiblesse du moi qui cherche refuge auprès de l'autorité, tendances à l'agression contre l'autre, et tendances parallèles à l'autodestruction.

De ce constat, Horkheimer tire la conclusion que les pratiques sociales et politiques peuvent receler de forts potentiels régressifs, et qu'il ne peut y avoir d'émancipation si elles ne sont combattues avec la plus grande lucidité. Or, force est de se rendre compte que ce type de problèmes n'a jamais été pris en charge par les marxistes, ce qui n'a pas manqué de se faire sentir dans les actions collectives et les formes d'organisation du mouvement ouvrier. Ce der- nier, en réalité, n'a pu faire face à la montée du nazisme parce qu'il a souvent joué, bien que dans une moindre mesure, sur les mêmes mécanismes régressifs que les nazis.

5 À la fin des années trente, et surtout au cours des années quarante, Horkheimer et son ami Adorno, profondément frappés par les persécutions antisémites en Allemagne, puis par la politique d'extermination des juifs d'Europe, vont mettre en question non seulement les tendances à la barbarie présentes dans les relations sociales, mais aussi toute l'évolution de la culture occidentale mythifiant la raison instrumentale et le progrès économique. Dans La Dialectique de la raison, œuvre la plus signifiante de cette période, la rupture avec le marxisme est complète. Pourtant, contrairement à ce qui est sou- vent affirmé - voir par exemple Habermas - cela n'implique pas l'abandon de tout projet d'émancipation. Ce que Horkheimer et Adorno entendent faire, c'est pousser jusqu'au bout l'analyse des difficultés, voire des quasi-impossibilités des luttes pour la libération. C'est pourquoi La Dialectique de la raison s'attache à cerner les obstacles à combattre, en particulier ce que Horkheimer et Adorno appellent l'industrie culturelle (essentiellement les médias) et qui finit par peser d'un poids énorme sur les processus cognitifs, la culture savante et les activités artistiques. Par là, la culture tautologique est aveugle, elle ne fait plus que redoubler et confirmer les formes de domination.

6 Ces vues pessimistes expliquent fort bien que Horkheimer et Adorno ne partagent pas l'euphorie générale après la défaite de l'Allemagne nazie. Ils se réjouissent certes de voir fini le cauchemar, mais ils sont persuadés que la démocratie confirmée ou 
restaurée dans de nombreux pays est une plante fragile. Pour eux, ce qui s'est passé en Allemagne peut très bien se renouveler ailleurs sous d'autres formes, et l'avenir reste forcément incertain. C'est dans ce contexte qu'il faut paradoxalement replacer le retour en Allemagne. Il s'agit d'un pays traumatisé par l'épisode nazi et la perte de son indépendance, et encore relativement épargné par la croissance de l'industrie culturelle. Il apparait donc comme un champ d'expérience possible pour les tenants d'une théorie critique. On peut y tester les séquelles sur les individus et les groupes sociaux d'une période de barbarie sans précédent en Europe, on peut y tester aussi les effets d'une démocratie octroyée par des puissances occupantes. La refondation de l'Institut de recherche en science sociale de Francfort s'inscrit donc dans un cadre très particulier, elle n'est pas pure et simple continuation de ce qui se faisait avant 1933, mais mise au point de nouveaux dispositifs intellectuels. Il s'agit pour la théorie critique d'arriver à survivre et à se développer, sans pour autant se référer à un quelconque agent historique de la transformation sociale ou à un quelconque garant de la réussite de l'émancipation. Il lui faut être à elle-même sa propre pratique, en s'affirmant dans le champ intellectuel. Cela ne peut évidemment se faire par le seul travail d'intellectuels solitaires assis devant leur machine à écrire, cela exige en fait des activités collectives de recherche, des activités de diffusion des résultats obtenus, des controverses scientifiques. La théorie critique doit se mettre à l'épreuve, se mesurer avec une réalité, sinon hostile, du moins peu réceptive, pour produire des effets. Elle ne doit pas chercher à dire en positif ce que sont le monde et la société, mais doit faire apparaître tout ce qui contredit et perturbe les dispositifs sociaux dominants.

7 Il ne peut être question, dans cet esprit, de bâtir une théorie totalisante de la société, car ce serait supposer qu'il y a harmonie entre le général et le parti- culier, et ce serait nier les discontinuités et les médiations brisées entre les rap- ports sociaux et les individus. Les pratiques de recherche et les pratiques de théorisation doivent en conséquence être des pratiques qui prennent à revers les agencements théoriques dominants et les déséquilibres en permanence. En aucun cas la sociologie critique ne peut prendre les choses de haut et s'opposer abstraitement aux différents courants sociologiques. Sa tâche, au contraire, est de se glisser dans leurs problématiques pour en montrer les limites, les inconsistances et l'acceptation plus ou moins masquée de la fausse totalité que constitue l'ensemble des rapports sociaux. Il va de soi que des considérations du même ordre peuvent être faites à propos des méthodes et des techniques de recherche. Il faut en particulier pratiquer la critique interne de la recherche empirique, et produire à partir de là de nouveaux instruments de connaissance qui permettent de restituer toutes les médiations entre singulier, particulier et général, occultées par la domination totalisante du général, l'accumulation du capital et la gestion étatique. Cela ne permettra certes pas la possibilité de connaître le tout social, mais cela fera surgir des problèmes ignorés, des points de jonction et de friction entre le général et le particulier, ainsi que des ruptures de continuité dans la trame sociale apparemment lisse. Aussi, pour rester fidèle à cette orientation, la sociologie critique doit-elle à tout prix éviter le piège du méthodologisme, c'est-à-dire les méthodes et techniques qui gomment les aspérités du social. Il faut au contraire, comme dans l'enquête sur la personnalité autoritaire effectuée aux États-Unis, avoir une approche méthodologique multilatérale, qui organise en constellation des techniques multiples.

8 Si le but recherché par Horkheimer et Adorno n'est pas une théorie générale de la société, il n'est pas non plus l'accumulation de théories de niveau intermédiaire à partir 
de généralisations limitées. Ce qu'ils ont en vue, ce sont des déconstructions théoriques et des démontages de l'empyrée, qui mettent à mal l'immanence totale (la fermeture de la société sur elle-même), c'est-à-dire l'organisation symbolique du monde à partir des automatismes de la marchandisation et de la valorisation. Cette perspective s'est naturellement heur- tée à beaucoup d'oppositions, et cela d'autant plus que l'Institut, ayant acquis beaucoup d'expérience professionnelle aux États-Unis, jouissait d'un grand prestige. On peut parler à ce propos d'une sorte de guerre civile larvée dans le monde de la sociologie. L'Institut est craint parce qu'il associe, de façon déroutante pour beaucoup, une réflexion théorique de haut niveau et un intense travail de terrain. On ne peut l'accuser ni de théoricisme ni d'empirisme, et de nombreuses attaques vont avoir des connotations idéologiques. Certains reprochent à l'Institut d'introduire le marxisme en contrebande dans la culture sociologique, d'autres au contraire, influencés par le matérialisme historique des pays de l'Est, critiquent sa révision, voire son abandon du marxisme. D'autres encore lui font grief d'être une sorte de corps étranger dans la sociologie allemande, en raison de son passé dans l'émigration. Mais toutes ces attaques faites dans les années cinquante et soixante n'ont pas vraiment porté ombrage à l'Institut, elles ont plutôt eu pour conséquence d'attirer l'attention sur lui. Adorno devient président de la société allemande de sociologie, ce qui lui donne l'occasion d'être l'initiateur de deux débats très importants sur « capitalisme tardif ou société industrielle » et sur « les méthodes en sciences sociales », qui trouvent un très grand écho. Sans être hégémonique, l'Institut exerce une influence considérable encore renforcée par la qualité de ses publications, par son implantation dans le monde étudiant.

Comme le dit Adorno, l'Institut, avec tous ceux qui l'entourent, agit un peu comme un parti dans le monde intellectuel. L'équipe réunie autour de Horkheimer et Adorno attire beaucoup de monde et contribue à former de nouvelles générations de sociologues. Les interventions dans la presse, à la radio, dans les revues de Horkheimer et Adorno, la publication de leurs livres, sont souvent commentées et donnent lieu à des échanges polémiques. Ils bénéficient aussi du fait qu'ils ne sont pas étroitement sociologues, mais interviennent dans les domaines de la philosophie ou de l'esthétique, en récusant les spécialisations. Ce faisant, ils ne cherchent pas à s'éloigner de la théorie de la société ; tout au contraire, ils entendent bien lui donner plus d'ampleur et de profondeur, en s'attaquant aux frontières de la division intellectuelle du travail et aux fétichismes sectoriels dans les processus cognitifs. L'autarcie de la philosophie, comme celles de l'esthétique et de la sociologie sont en fait porteuses d'hétéronomie. Par contre, c'est en multipliant les contacts entre les différentes sphères que l'on peut produire de l'autonomie et combattre de l'idéologie en tant que ciment du fonctionnement ordinaire du système.

10 Cependant, ce travail de dévoilement pratico-théorique, qui a eu en définitive plus de succès que ne l'espéraient ses initiateurs au départ, a connu beau- coup de malentendus. Horkheimer et Adorno en sont en partie responsables, ayant par exemple pratiqué l'autocensure au niveau de la terminologie, afin d'éviter d'être confondus avec les différentes orthodoxies marxistes. Mais c'est surtout la nouveauté qui a dérouté bien des esprits, et l'histoire de la réception des théories francfortiennes est faite de beaucoup d'incompréhensions et d'interprétations erronées. Très souvent, on perçoit la théorie critique comme une sorte de philosophie négativiste, qui ne voit dans la sociologie qu'un moyen d'illustrer son pessimisme quant à l'évolution de la société. C'est évidemment ignorer que, loin de toute délectation morose et résignée, 
Horkheimer et Adorno - et surtout Adorno - se sont efforcés de construire une théorie de la société qui soit en décalage par rapport à la société contemporaine, et donc lui renvoie une image qu'elle n'a pas d'elle-même, mais une image vraie.

11 La sociologie, selon eux, ne saurait être immobiliste, elle doit même produire, outre des changements dans le domaine intellectuel, des déséquilibres dans les rapports sociaux, seraient-ils infimes. La sociologie ne doit pas enregistrer, elle doit se faire intervention, même s'il s'agit d'interventions limitées. Étudier les processus de formation, c'est aussi s'interroger, non seulement sur ce qui se fait, mais sur les modifications - les négations déterminées - dans les processus d'apprentissage qui peuvent influer sur les processus cognitifs. S'intéresser à la formation politique dispensée dans les universités populaires, ce n'est pas seulement peser des programmes et des contenus, c'est aussi suivre les transformations des conceptions de la politique chez ceux qui l'étudient.

Il est vrai que Horkheimer et Adorno ont beaucoup critiqué l'activisme, notamment l'activisme du mouvement étudiant de la fin des années soixante et de tous ceux qui, dans cette période, préconisaient un changement global des rapports sociaux. Pourtant, il faut bien voir que cette attitude critique ne relève pas, comme le pensent beaucoup, de positions conservatrices, voire d'une proscription de la politique. En réalité, on est en présence d'un attentisme dicté par la crainte que les actions collectives ne soient contaminées par les pratiques du communisme d'inspiration soviétique. Toutefois, il ne s'agit pas d'un attentisme quiétiste, il s'agit d'un attentisme inquiet qui espère bien que le dernier mot n'aura pas été dit.

\section{INDEX}

Mots-clés : Théorie critique, Ecole de Francfort, lutte des classes, Max Horkheimer, Erich Fromm

\section{AUTEUR}

\section{JEAN-MARIE VINCENT}

Jean-Marie Vincent fut le fondateur de la revue Variations 